\title{
The Application Mode and Effectiveness of Playful Positive Psychology Course with Six Bricks and DUPLO® Play Box for High School Students
}

\author{
Pay-Ling Harn
}

\begin{abstract}
This study was aimed at using the playful approach of Six Bricks and DUPLO ${ }^{\circledR}$ Play Box (SBPB) to develop the playful positive psychology course (PPPC) for Taiwan high school students. 21 Taiwanese high school students were invited to attend 2-hour SBPB PPPC, including 14 male students and 7 female students. The core curriculum content was based on the broaden-and-build theory of positive emotions. The "positivity-ratio scale" developed by Fredrickson was used to conduct pre-test and post-test. Paired-sample $t$ test was adopted to evaluate the impact of SBPB PPPC on the positive emotions, negative emotions and positivity-ratios of high school students. The findings of this study were as follows: (1) positive emotions and positivity-rations exhibited upward trends, whereas negative emotions presented a declining trend. The positivity-ratio is from 1.79 to 13.54 . After participating in the course, it is converted from below positivity-ratio 3 to above positivity-ratio 3. (2) The score of positive emotions, negative emotions, positivity-ratio achieved significant immediate effect.

SBPB PPPC has the effect of promoting positive emotional adaptation for high school students. Based on the results of the study, suggestions are made for the subsequent use of Six Bricks and DUPLO ${ }^{\circledR}$ Play Box in the adolescents' curriculum.
\end{abstract}

Index Terms - playful approach, positive psychology course, broaden-and-build theory, and high school students, Six Bricks, DUPLO ${ }^{\circledR}$ Play Box.

\section{INTRODUCTION}

Fredrickson [2] proposed the broaden-and-build theory of positive emotions to describe the importance of positive emotions. The theory posits that positive emotions can broaden people's momentary thought-action repertoires, which in turn serves to build their enduring personal resources for developing response strategies to survive in the face of life shocks. Positive emotions can eliminate the negative effects of negative emotions, a phenomenon called the undo hypothesis [1]. According to broaden-and-build theory, if the students' positive emotions are triggered first in the course, it will help expand the thinking of learning. How to stimulate more positive emotions in the teaching process? In recent years, at the practice site of teaching, the playful learning modes have begun to be integrated. If we can stimulate positive emotions by "play", it will help expand the learning effect of students. Among them, Six Bricks and DUPLO $^{\circledR}$ Play Box ${ }^{\circledR}$ are two kinds of playful learning

Pay-Ling Harn, Assistant Professor, Applied Psychology Department, Hsuan Chuang University, No.48, Hsuan Chuang Rd., Hsiang San District, Hsinchu City, Taiwan methods that have attracted much attention from educators in recent years.

Six Bricks and DUPLO® Play Box (abbreviated as SBPB) were developed by a group of South African teachers who hope to promote early childhood development and better subsequent life-long learning. These two playful learning methods take a holistic approach, emphasizing the importance of physical, cognitive, creative, social, and emotional skills, and how these skills influence and support each other. SBPB trigger the playful learning characteristics of joyful, actively engaging, meaningful, iterative, and social interactive [10]. Joyful, actively engaging, meaningful, and socially interactive correspond directly to the four elements of the PERMA model [9], that is, positive emotions, engagement, relationship, and meaning. In addition, problem solving and knowledge transfer during the iterative process can lead to achievements. This also illustrates the correlation between the psychological change mechanism of SBPB and positive psychological promotion. In addition, SBPB emphasize the importance of exercise for learning. Exercise awakens the brain to learn and allows the entire body to collect information through the senses [10].

Six Bricks and DUPLO® Play Box were originally used for children's education. In recent years, it has begun to expand to learning models of different ages and topics. In cognitive development, children and adolescents are divided into concrete operational stage and formal operational stage, which are two different learning forms. Therefore, the researcher wants to explore how SBPB's playful learning model can be applied to the teenagers' curriculum? Relevant research has also pointed out that Six Bricks and DUPLO ${ }^{\circledR}$ Play Box have demonstrated functions such as regulating anxiety, promoting positive emotions, improving emotional regulation, developing interpersonal cooperation, and social connections [5], [6], [7]. Adolescents face a critical transition period of physiology and psychology, which is a period of emotional storm during the development stage [8]. Failure to have good emotional adjustment will easily affect their life and learning adaptation.

Fredrickson [3] illustrated that the existence of both positive and negative emotions has its importance, and that the individual's positive and negative emotions also have the most appropriate ratio. The ratio of positive emotions to negative emotions is called the positivity-ratio or Losada-ratio. If the positivity-ratio is greater than three to one, the individual can have more well-being, which also improves its positive adaptation. It has also been confirmed in 
previous studies that individuals with well-being have positive ratios greater than or equal to 3 [5], [6], [7]. Therefore, the researcher also wants to further explore whether it is possible to increase the positivity-ratio of teenagers by SBPB?

Therefore, this study adopts playful learning methods of Six Bricks and DUPLO ${ }^{\circledR}$ Play Box, and uses the broaden-and-build theory as the teaching strategy and core content to develop the 2-hour playful positive psychology course (PPPC) and further explore the effects of SBPB PPPC's on teenagers' emotions. As stated above, the purposes of this study are as follows:

1. What is the application model of SBPB PPPC?

2. What is the effectiveness of SBPB PPPC on adolescents' emotional adaptation?

\section{METHOD}

A. Playful Positive Psychology Course with Six Bricks and DUPLO® Play Box (SBPB PPPC)

SBPB PPPC is 2 hours. The course will be divided into three parts: brain activation, positive emotion initiation, and core course learning. The curriculum structure of SBPB PPPC is shown in Table 1.

Table 1. Playful positive psychology course with SBPB

\begin{tabular}{ll}
\hline Stage & Learning activities \\
\hline Brain activation & Balance exercises with Six \\
& Bricks \\
Positive emotion & Learning activity of Tricky \\
& Tower, Back to Back with \\
initiation & Six Bricks
\end{tabular}

core course learning

Watch the "gratitude of
nature" video, learn the
classification of positive
emotions with the SBPB
positive emotion card,
understand the
broaden-and-build theory,
use the Play Box to
construct positive emotion
symbols, and use Six Bricks
to build gratitude trees

Six Bricks and DUPLO® Play Box originated in South African children's education practices. When it is used in other regions or ethnic groups, it must be adjusted in consideration of the learner's past learning experience and background. Joy is the core of SBPB. Joy may come from the fun of the learning activity itself, or it can successfully challenge the learning task. As far as researchers' experience of using Six Bricks and DUPLO ${ }^{\circledR}$ Play Box in Asia, the Asian learning model sometimes emphasizes the inner meaning connection rather than pure joy or creativity, which is also an important key to promote their long-term learning motivation.

Children belong to concrete operational stage [8]. Six Bricks and DUPLO ${ }^{\circledR}$ Play Box is an operational, concrete, and experiential learning model that allows children to learn through substantial experience. Adolescents enter the formal operation stage [8]. They have developed the cognitive models that use concepts to generalize and analogize knowledge. In this study, the researcher will use the Six Bricks and DUPLO ${ }^{\circledR}$ Play Box as a way to stimulate the breadth and depth of adolescent's formal operations. Therefore, in this study, SBPB will be used to activate the brain and stimulate positive emotions to promote deep learning of core curriculum content. The inner joyful learning process will be triggered through playful learning and

deepening

learning.

In the first stage, lead high school students to perform Six Bricks balance exercise. This is based on the importance of exercise for learning, which can activate the brain and promote the operation of the brain mechanism of learning. In the second stage, it is the use of "Tricky Tower" (Figure 1) and "Back to Back "(Figure 2) with Six Bricks to promote high school students' joy, engagement, and social interaction. In the second stage, it can be observed that the high school is full of engagement and joyful emotions. During the Tricky Tower, the high school students continue to challenge more difficult task activities by themselves.

The third stage is the core course study. Through the watching of the "gratitude of nature" video, it helps high school students to sense their positive emotions. The SBPB positive emotion card compiled by the researchers is used to guide high school students to recognize the ten positive emotions proposed by Fredrickson and further identify the positive emotions of the self. Next, the positive emotion symbol at this moment (Figure 3) is constructed by the play box to visualize the positive emotion feeling. Group as a unit, use six bricks to construct a gratitude tree (Figure 4) to deepen the understanding of positive emotions, and combine the positive emotion symbol and gratitude tree to integrate the learning of the course. Next, the researchers briefly described broaden-and-build theory, and led high school students to play box to build the symbol of positive emotions at this moment (Figure 4), to visualize the positive emotions. Take the group as a unit, use six bricks to construct the gratitude tree to deepen the understanding of gratitude, and combine the positive emotion symbol with the six bricks gratitude tree (Figure 4) to unify the course learning.

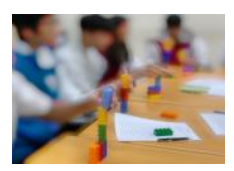

Figure1. Tricky tower

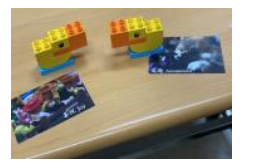

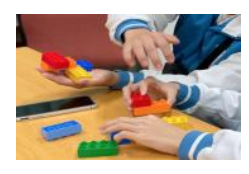

Figure2. Back to back.

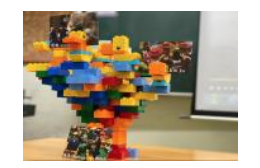

Figure3. Emotional symbols Figure4. Gratitude tree

\section{B. Participants}

The participants of this study were 21 high school students, aged 17 to 19 years, including 14 boys and 7 girls. 


\section{Research Instruments}

(a) Positivity-ratio scale. In this study, Fredrickson [2] " Positivity-ratio scale " was used to measure the participants' positive emotions, negative emotions, and positivity-ratio. There are 20 questions on the scale, including positive emotions (10 items) and negative emotions (10 items). Designed with Likert five-point scale, the scoring is from 0 to 4. A higher score indicates a higher positive or negative emotion. The positivity-ratio is calculated by adding up the scores of items with a positive emotion score greater than 2, adding up the scores with a negative emotion score greater than 1 , and dividing the above two total scores to calculate a positivity-ratio.

(b) SBPB positive emotion card. The researcher developed the SBPB positive emotion card. Use the images of Six Bricks or DUPLO ${ }^{\circledR}$ Play Box to compile ten positive emotion cards proposed by Fredrickson [2], including joy, gratitude, serenity, interest, hope, pride, amusement, inspiration, awe, love. The front of the card is the name and image of positive emotions, and the back is the definition of positive emotions (Figure 5 and 6).

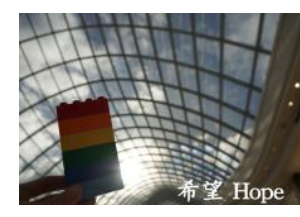

Figure5. the front of card

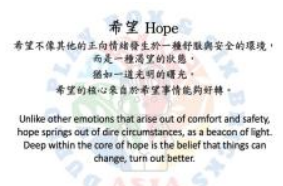

Figure6. the back of card

\section{Date Analysis}

Before and after the course, 21 research participants completed the pre-test and post-test of the " Positivity-ratio scale ". The researcher used the dependent sample $t$ test to make a difference comparison to explore the effect of SBPB PPPC on adolescents' positive emotions, negative emotions, and positivity-ratios.

\section{RESULT}

Before and after the course, the research participants will fill in the "positive ratio scale" to understand the status and changes of their positivity-ratios. The researchers calculated the average score of 10 positive emotion items and 10 negative emotion items respectively. In addition, the positivity-ratio is calculated according to Fredrickson's definitions. First, the study calculates the average score of positive emotions, negative emotions, and positivity-ratio to explore the changing trend of different dimensions before and after the course.

It can be seen from figure 7 that the research participants participating in SBPB PPPC showed a upward trend in positive emotions and a declined trend in negative emotions. It can be seen from figure 8 that after participating in the course, the research participants' positivity-ratios jumped from lower than " 3 " to higher than " 3 ". According to previous research (Fredrickson, 2009, 2013; Fredrickson \& Losada, 2005), individuals with happiness and well-being have positive ratios greater than 3. Therefore, after participating in SBPB PPPC, the ratios of positive emotions to negative emotions of high school students reach an ideal state.

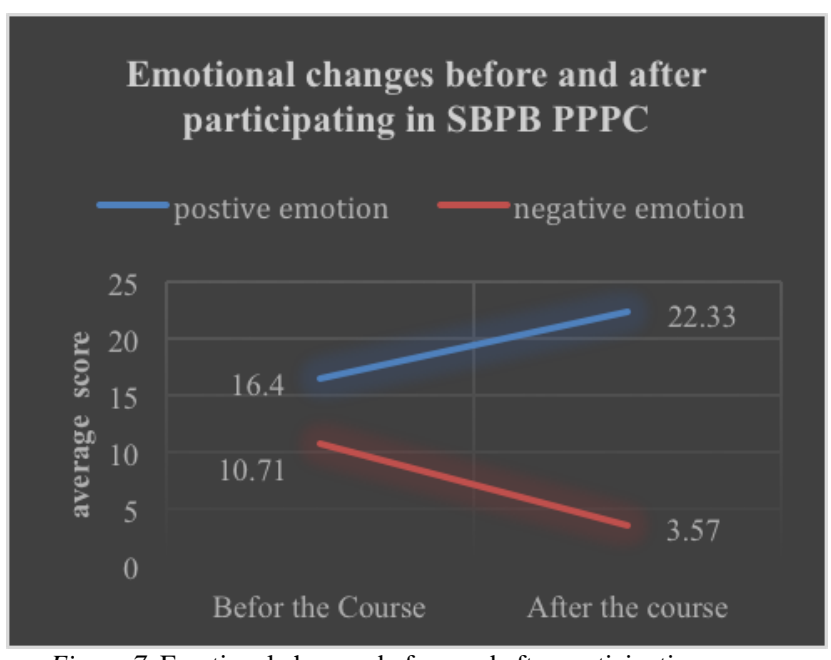

Figure 7. Emotional changes before and after participating course

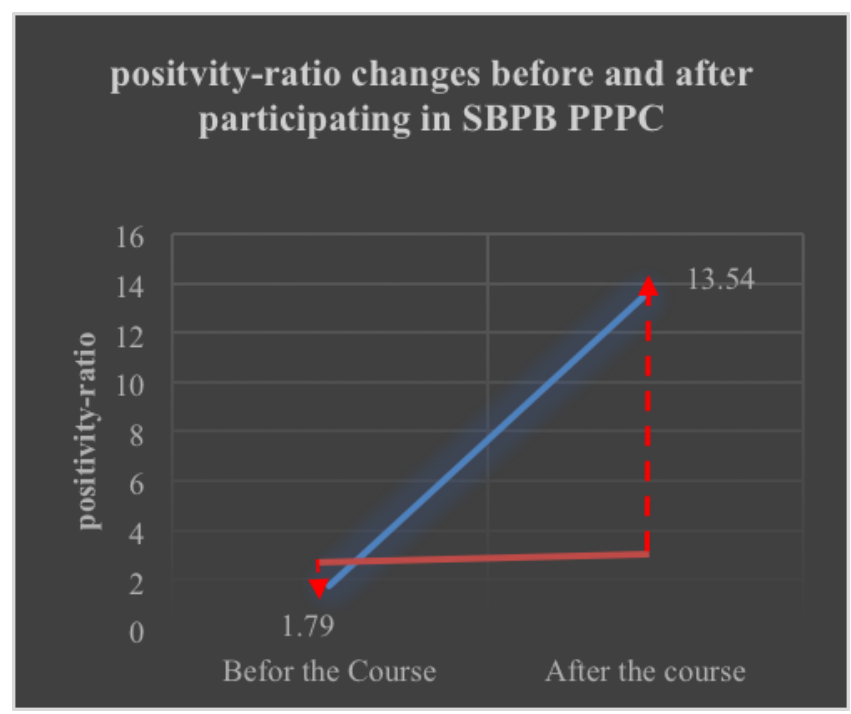

Figure 8. Positivity-Ratio changes before and after participating course

The researcher further compared the differences in the averages by using Paired-sample $t$ test to explore the effect of SBPB PPPI on the emotions of the research participants. As can be seen from Table 2, after participating in the positive psychology course, the scores of positive emotions $(t=-3.43$, $p<.05)$, negative emotions $(t=5.56, p<.01)$ and positivity-ratios $(t=-6.46, p<.01)$ all achieved significant immediate effects.

Table 2. Dependent samples t-test analysis table $(\mathrm{N}=21)$

\begin{tabular}{ccccccc}
\hline Variable & \multicolumn{2}{c}{ Pre-test } & \multicolumn{2}{c}{ Post-test } & $t$ & $p$ \\
& $\mathrm{M}$ & $\mathrm{SD}$ & $\mathrm{M}$ & $\mathrm{SD}$ & $d f=20$ & \\
\hline $\begin{array}{c}\text { Positive } \\
\text { emotion }\end{array}$ & 16.40 & 8.22 & 22.33 & 7.03 & $-3.43^{*}$ & .003 \\
\hline $\begin{array}{c}\text { Negative } \\
\text { emotion }\end{array}$ & 10.71 & 7.82 & 3.57 & 7.13 & $5.56^{* *}$ & .000 \\
\hline $\begin{array}{c}\text { Positivity } \\
\text {-ratio }\end{array}$ & 1.79 & 1.20 & 13.54 & 8.65 & $-6.46^{* *}$ & .000 \\
\hline
\end{tabular}

\section{COUNCLUSION}

This research is to explore the application model of Six Bricks and DUPLO® Play Box for adolescent courses, which was originally used in children's education The researcher adjusted the children's education model of Six Bricks and 
DUPLO® Play Box based on Asian learning models and adolescent's development characteristics. The course design uses the SBPB's playful characteristics to promote the activation of brain learning and positive emotions. In addition, researchers have used the learning mechanism of the broaden-and-build theory [3] to design the playful positive psychology course. According to the course observation, in the early part of the course, playful learning activities of SBPB were the main axis. Adolescents are fully engaged to the atmosphere of playful learning, which highly triggers positive emotions and joyful social interactions. According to the results of the study, SBPB PPPC has achieved significant results in positive emotions, negative emotions and positivity-ratios. The results of this study echo the researchers' observations. The results of this study are also consistent with previous studies [5], [6], [7]. Six Bricks and DUPLO ${ }^{\circledR}$ Play Box can promote positive emotions and mediate negative emotions and social connection.

According to the teaching concept of using the broaden-and-build theory as a strategy, the initiation of positive emotions is indeed achieved in the early stages of the course. The purpose of watching "gratitude of nature" video is to stimulate high school students to have more different positive emotions (for example, a student said that he started from joy, inspired to serenity), and used to explain the connotation of the broaden-and-build theory. In reflective discussions, students can extend gratitude at this moment (grateful for participating in this course) to gratitude in life, which also shows the flexibility and extension of high school students' learning thinking [2], [3]. Finally, deepen learning by constructing positive emotion symbols with Play Box and gratitude trees with Six Bricks. Due to the characteristics of adolescents' formal thinking, adolescents can use metaphors to explain the models they construct, and they will form the concepts learned in the classroom.

The study was designed to be a single 2-hour session, which would limit the applicability of long-term courses and the inference of research results. However, this study provides an innovative positive psychology curriculum structure for adolescents that incorporates Six Bricks and DUPLO® Play Box. Integrate the learning mechanism of positive psychology and the psychological characteristics of playful learning to assist young people in the study of concepts and theories. Both course observation and quantitative research have shown good results, not only can indeed promote a good adaptation of high school students, but also and demonstrate good learning motivation and understanding. Therefore, this study still has reference value for the follow-up adolescents' positive psychology course. Later, the concept of curriculum design in this research can be used as a prototype to develop a long-term curriculum. In this study, only measures emotional adaptation. For the evaluation of learning effects, in addition to the teacher's observations of students' learning motivations, assessments of learning motivation and learning achievements can be included in subsequent studies.

\section{REFERENCES}

[1] Fredrickson, B.L., \& Levenson, R.W. (1998). Positive emotions speed recovery from the cardiovascular sequel of negative emotions. Cognition and Emotion, 12,191-220.

[2] Fredrickson, B. L. (2009). Positivity. New York: Three rivers press.
[3] Fredrickson, B. L. (2013). Updated thinking on the positivity ratio. American Psychologist, 68, 814-822.

[4] Fredrickson, B. L., \& Losada, M. (2005). Positive affect and the complex dynamics of human flourishing. American Psychologist, 60 (7), 678-686.

[5] Harn, P. L., \& Bo, S.H. (2019). The effectiveness of playful positive psychology interventions with Six Bricks and DUPLO® Play Box for Taiwan children on emotional adaptation. World Journal of Research and Review, 9(5), 5-8. doi: 10.31871/WJRR.9.5.4

[6] Harn, P. L., \& Hsiao, C.C. (2018a). A Preliminary Study on LEGO®-Based Workplace Stress Reduction with Six Bricks and LEGO® SERIOUS PLAY® in Taiwan. World Journal of Research and Review, 6(1), 64-67.

[7] Harn, P. L., \& Hsiao, C.C. (2018b). Strenghth-4D career model with LEGO® Serious Play and Six Bricks. International Journal of Management and Applied Research, 5 (4), 157-172. doi: 10.18646/2056.54.18-012

[8] Kail, R.V., \& Cavanaugh, J. C. (2016).Human development: A life-span view. Singapore: Cengage Learning

[9] Seligman, M.E.P. (2011). Flouring: A new understanding of happiness and wellbeing. New York, NY: Nicholas Berkley Publishing.

[10] Zosh, J.M., Hopkins, E. J., Jensen, H., Liu, C., Neale, D., Hirsh-Pasek, K., Solis L.S., \& Whitebread, D. (2017). Learning through play: a review of the evidence. Demark: LEGO Foundation.

Pay-Ling Harn received Ph.D. degree in educational psychology and counseling from Taiwan Normal University, Taipei, Taiwan. Currently, she is an assistant professor at Hsuan Chuang University, Hsinchu, Taiwan. She is certified counseling psychologist, Six Bricks and Play Box master trainer. Her research interests are in positive psychology, career counseling, and playful positive psychology interventions. 